\title{
Disparities in Injury Mortality Between Uganda and the United States: Comparative Analysis of a Neglected Disease
}

\author{
Sudha Jayaraman $\cdot$ Doruk Ozgediz $\cdot$ Justin Miyamoto $\cdot$ \\ Nolan Caldwell • Michael S. Lipnick • Cephas Mijumbi • \\ Jacqueline Mabweijano $\cdot$ Renee Hsia $\cdot$ Rochelle Dicker
}

Published online: 23 December 2010

(c) The Author(s) 2010. This article is published with open access at Springerlink.com

\begin{abstract}
Background The burden of global injury-related deaths predominantly affects developing countries, which have little infrastructure to evaluate these disparities. We describe injury-related mortality patterns in Kampala, Uganda and compare them with data from the United States and San Francisco (SF), California.

Methods We created a database in Kampala of deaths recorded by the City Mortuary, the Mulago Hospital
\end{abstract}

S. Jayaraman $(\bowtie) \cdot$ R. Dicker

Department of Surgery, University of California San Francisco, 513 Parnassus Avenue, S321, San Francisco, CA 94118, USA e-mail: sudhapjay@gmail.com

\section{Ozgediz}

Division of Pediatric Surgery, Hospital for Sick Children, University of Toronto, 555 University Avenue,

Toronto, ON M5G 1X8, Canada

\section{J. Miyamoto · N. Caldwell}

School of Medicine, Office of Medical Education, University of California San Francisco, San Francisco, CA, USA

M. S. Lipnick

Department of Medicine, Brigham and Women's Hospital,

75 Francis Street, Boston, MA 02115, USA

C. Mijumbi

Department of Anesthesia, Mulago Hospital and Makerere University, Kampala, Uganda

J. Mabweijano

Department of Accident and Emergency,

Mulago Hospital and Makerere University, Kampala, Uganda

R. Hsia

Department of Emergency Medicine, University of California

San Francisco, 505 Parnassus Avenue, Room M-24,

San Francisco, CA 94143, USA
Mortuary, and the Uganda Ministry of Health from July to December 2007. We analyzed the rate and odds ratios and compared them to data from the U.S. Centers for Disease Control and Prevention and the California Department of Public Health.

Results In Kampala, $25 \%$ of all deaths were due to injuries (812/3303) versus $6 \%$ in SF and 7\% in the United States. The odds of dying of injury in Kampala were 5.0 times higher than in SF and 4.2 times higher than in the United States. Age-standardized death rates indicate a 93\% greater risk of dying from injury in Kampala than in SF. The mean age was lower in Kampala than in SF (29 vs. 44 years). The adult injury death rate (rate ratio, or RR) was higher in Kampala than in SF (2.3) or the United States (1.5). Head/neck injury was reported in $65 \%$ of injury deaths in Kampala compared to $34 \%$ in SF [odds ratio (OR) 3.7] and $28 \%$ in the US (OR 4.8).

Conclusions Urban injury-related mortality is significantly higher in Uganda than in the United States. Injury preferentially affects adults in the prime of their economically productive years. These findings serve as a call for stronger injury prevention and control policies in Uganda.

\section{Introduction}

More than five million people worldwide die from injury annually [1]. Disability from injury affects an additional 20-50 million people each year [2]. Poor people in individual countries are particularly affected by injury and bear an inequitable distribution of the burden of injury [3]. The Global Burden of Disease Project now predicts that the global burden of injury will increase by $28 \%$ between 2004 and 2030, whereas major communicable illnesses such as tuberculosis, malaria, and human immunodeficiency virus 
infection/acquired immunodeficiency syndrome (HIV/ AIDS) will decline during the same time [4]. The new Global Burden of Disease Injury Expert Group has recently launched a call for data from existing local, regional, and national sources around the world to generate an accurate estimate of the global burden of injury [5].

The public health and donor communities have recently used health disparities as a measure of global health equity, but there are few data available from low- and middleincome countries to measure the disparity in injury-related mortality and morbidity or to compare with data from resource-rich settings [5-7]. Currently, 90\% of all deaths from road traffic injuries occur in low- and middle-income countries [8]. Injury mortality has been shown to vary by context, including economic level; for example, in 1998 injury-related mortality was as high as $63 \%$ in Kumasi, Ghana but was 55\% in Monterrey, Mexico and 35\% in Seattle, Washington, USA [9]. Despite these findings, many low- and middle-income countries have disparate data sources that do not allow rigorous analysis of injury disparities. Few have collated data from these existing systems to describe a comprehensive picture of injury [6].

In sub-Saharan Africa, the need for improved vital statistics is well known [10]. For example, according to the International Institute for Vital Registration and Statistics, only $20 \%$ of deaths in Ghana are registered with the government. Existing injury surveillance data collection systems in Ghana suggest that $80 \%$ of deaths occur outside the hospital and that police records in Ghana grossly underrecord prehospital injury mortality [11]. Additionally, only $31 \%$ of severely injured people are thought to access health facilities, which further limits the quality of data from hospital-based trauma registries [12].

In Uganda, where injury is a growing problem, a 1996 Burden of Disease study identified injury as one of the top six causes of death in half of Uganda's districts. Since 1996, the injury burden has been assessed through hospitalbased trauma registries and data collection through the Uganda National Household Survey, although these measures probably underestimate injury burden from high prehospital mortality and poor access to emergency services [10-12]. Few data on injury mortality rates are available from Kampala. Furthermore, there is no information on how these rates compare to those in resourcerich settings.

In this study, we hypothesized that injury mortality is high in urban Kampala and that rates are higher than those from an industrialized city in a high-income country (San Francisco, California, USA). To investigate this hypothesis, we created a mortality database in Kampala using data from three main sources and compared the findings from this database with data from the Centers for Disease Control and Prevention (CDC) and San Francisco County.

\section{Materials and methods}

\section{Study sites}

Kampala, Uganda and San Francisco, California were selected based on an ongoing academic collaboration between the Departments of Surgery, Anesthesia, and Accident \& Emergency at Mulago Hospital/Makerere University and the University of California San Francisco.

Uganda and the United States represent different economic levels and health care settings. During 2006-2007, Uganda had a population of $29,899,000$ and a gross national per-capita income of $\$ 370$. The total per-capita health expenditure was $\$ 25$, and the life expectancy was 51 years for women and 49 years for men. At the same time, the United States had a population of $302,841,000$ and a gross national per-capita income of $\$ 46,040$. The U.S. per-capita health expenditure was $\$ 6714$, and the life expectancy in the US was 80 years for women and 75 for men $[13,14]$. Kampala is the capital city of Uganda, with 1,208,544 residents (2002 census data) [15]. Mulago National Referral Hospital is the sole 24-hour public emergency department in Kampala and has the only postgraduate surgical training program in the country. Mulago receives approximately 4000 injured patients every year. San Francisco is a major city in the United States, with a population of 801,522 [16]. San Francisco General Hospital (SFGH) is the city's only Level 1 trauma center and is an academic institution affiliated with the University of California San Francisco. It has a full complement of specialist staff from neurosurgery, orthopedics, and general surgery; and it receives more than 3000 severely injured patients every year.

\section{Data sources in Kampala}

We compiled a comprehensive database of deaths recorded between July and December 2007 in Kampala using the three main sources of mortality data in the city: Mulago Hospital Mortuary, Kampala City Council Mortuary, and the Uganda Ministry of Health Postmortem Reports. We defined a 6-month time frame for data collection based on feasibility and selected the most recent time period for which data were available. The Mulago Hospital Mortuary records deaths for the sole public hospital in Kampala, which sees $75 \%$ of the injured victims in the city [17]. The records include data on demographics, date and mechanism of death, and findings at the time of death as noted by the treating clinician coded using the International Classification of Disease-10th Revision (ICD-10). The Kampala City Council Mortuary predominantly captures deaths that occur in the prehospital setting or at other hospitals and records data on demographics, date of death, last known address of victim, and 
detailed autopsy findings by the city's forensic pathologists. Ugandan culture discourages undocumented burials, so data from the two mortuaries are thought to capture most of the deaths in the city. The Ministry of Health collects postmortem reports from Mulago Hospital, the Kampala Police Department, and the Kampala City Council Mortuary regarding any deaths of a forensic nature. As such, the Ministry of Health records are not comprehensive. Nevertheless, we were able to use this data set to identify mechanisms of death and demographic data that were missing in some of the Mulago Hospital and Kampala City Council Mortuary records. We merged data from all three data sets and removed duplicates before proceeding with the analysis. We used the population noted in Ugandan census data during the study years as the population at risk.

Data sources in the United States

The CDC Web-based Injury Statistics Query and Reporting System (WISQARS) is a publicly available database that collects data from death certificates filed with states' vital statistics offices as reported to the CDC's National Center for Health Statistics [18]. Injuries are classified using the ICD-10 system. The system includes numbers of injury deaths in the United States and reports crude rates of injury by intent, cause, age, sex, race, and region/state. The system directly standardizes the data rates using the total U.S. population to report age-adjusted rates. The most recent data available are from 2005 and are based on a total population of $295,895,897$ residents.

\section{Data sources in San Francisco}

The San Francisco data are drawn from the California Department of Public Health [16] and the SFGH Trauma Registry. The Department of Public Health collects and compiles vital statistics data from county recorder's offices in the state of California and makes these records publicly available. The most complete data set available was from 2006. The SFGH Trauma Registry records all injured patients who receive care in the county's only Level 1 trauma center and uses the ICD-10 to classify injuries. The database provides information on age, mechanism, intent, body region injured, ICD-10 code, and injury severity.

Institutional Review Board approvals for this study were obtained from the University of California San Francisco, Mulago Hospital, and the Uganda National Council of Science and Technology.

Data management and analysis

The Ugandan data were entered into the 2003 Microsoft Excel program (Microsoft, Seattle, WA, USA). We categorized each death by five body regions - head and neck, chest, abdomen, extremities, unspecified/ trauma to multiple body regions - and analyzed injury deaths by demographics, mechanism, and body region most commonly affected. The age-specific rates from all three settings were applied to the World Health Organization's (WHO) standard population to obtain directly standardized rates and comparative mortality ratios [19]. Risks and rates were considered equivalent based on an assumption of a constant population at risk during the study period. We used Stata 2007 IC10 software (Stata, College Station, TX, USA) to calculate rate and odds ratios. We conducted significance testing using $\chi^{2}$ tests for all proportions and the $t$-test for the mean age of death.

\section{Results}

For the last 6 months of 2007, about $25 \%$ of the 3303 deaths recorded in Kampala were due to injury (Table 1). In contrast, $7 \%$ of deaths in the United States (US) in 2005 and $6 \%$ of deaths in San Francisco (SF) in 2006 were injury-related. These differences were statistically significant (Table 2). The odds of dying of injury in Kampala were 5.0 times greater than in SF and 4.2 times greater than in the US (Table 3).

\section{Distribution of injury deaths}

Among our data, 53\% of injury-related deaths in Kampala ( $n=434)$ occurred in the prehospital setting compared to $68 \%$ in SF $(n=243)$. Most of these prehospital deaths occurred in three main areas of the city (police precincts: Katwe, Jinja Road, Kira Road). Of the injury-related deaths at the hospital level, $94 \%$ of the deaths at Mulago Hospital

Table 1 Profile of deaths in the Kampala database

\begin{tabular}{lrr}
\hline Category & No. & $\%$ \\
\hline Total deaths $(n=3303)$ & & \\
Mulago Hospital mortuary & 2520 & 76.0 \\
Kampala City Council mortuary & 783 & 24.0 \\
Mechanism $(n=3303)$ & & \\
Injury & 812 & 25.0 \\
Noninjury & 2491 & 75.0 \\
HIV/AIDS & 602 & 18.0 \\
Tuberculosis & 314 & 9.5 \\
Malaria & 151 & 4.6 \\
Anemia & 138 & 4.0 \\
Opportunistic Infections & 24 & 0.7 \\
\hline
\end{tabular}

Categories are not exclusive. For example, a death could have been from both human immunodeficiency virus/acquired immunodeficiency syndrome (HIV/AIDS) and malaria and so would be captured in both categories in this table 
Table 2 Differences in injury mortality: Kampala, San Francisco, and the United States

\begin{tabular}{llll}
\hline Parameter & Kampala & SF & US \\
\hline $\begin{array}{l}\text { Percent of all deaths due to injury } \\
\text { Age at injury-related death }\end{array}$ & $25^{*}$ & 6.0 & 7.0 \\
$\quad$ (years), mean & $29^{*}$ & 44.0 & 47.0 \\
$\begin{array}{l}\text { Crude injury-related mortality } \\
\text { rate, per 10,000 }\end{array}$ & $6.7^{*}$ & 4.5 & 5.9 \\
$\quad$ Expected age-standardized mortality & $7.9^{*}$ & 4.1 & 5.5 \\
$\quad$ rate, per 10,000 (WHO population) & & & \\
Comparative mortality ratio & & & \\
$\quad$ Kampala vs. SF & $1.93^{*}$ & 1.0 & $\mathrm{n} / \mathrm{a}$ \\
$\quad$ Kampala vs. US & $1.44^{*}$ & $\mathrm{n} / \mathrm{a}$ & 1.0 \\
$\quad$ Injury-related mortality rate, per 10,000 & & & \\
$\quad$ Age $>19$ years & $11.0^{*}$ & 4.8 & 7.3 \\
$\quad$ Age 10-19 years & 2.4 & 3.6 & 3.0 \\
$\quad$ Age $<10$ years & $1.9^{*}$ & 2.0 & 1.2 \\
\hline
\end{tabular}

$S F$ San Francisco, US United States, $n / a$ not applicable

* Statistically significant difference: $p<0.01, \chi^{2}$ test

occurred in the inpatient population, rather than in patients in the emergency department. In contrast, at SFGH, $65 \%$ of all injury-related deaths were among inpatients (Table 4).

Injury deaths by demographic characteristics

In Kampala, $79 \%$ of injury-related deaths were in men compared to $69 \%$ in the US and $74 \%$ in SF. Most injuryrelated deaths in Kampala occurred in adults over 19 years of age ( $82 \%, n=628)$ (mean age 29 years, range $1-84$ years). In the Ugandan data set, age was broadly reported as adult, juvenile, or child in $40 \%$ of records and was missing in $6 \%$. The mean age was significantly lower in Kampala than in SF and the US (29 vs. 44 years in SF) (Table 3).

The injury mortality rate was significantly higher in adults in Kampala than in SF [rate ratio (RR) 2.3] or the US (RR 1.5). Mortality rates did not differ for the 10- to 19year age group across the three settings; however, in children $<10$ years of age, the injury mortality rate was significantly higher in Kampala and SF than in the US as a whole (2.0 vs. 1.2/10,000 children; RR 1.6). Age-

Table 3 Comparison of outcomes between Kampala, San Francisco, and United States

\begin{tabular}{lll}
\hline Outcome & $\begin{array}{l}\text { Kampala } \\
\text { vs. SF }\end{array}$ & $\begin{array}{l}\text { Kampala } \\
\text { vs. US }\end{array}$ \\
\hline $\begin{array}{l}\text { Odds of dying from injury } \\
\text { If dying of injury }\end{array}$ & 5.0 & 4.2 \\
Odds of having a head/neck injury & 3.6 & 4.7 \\
Odds of dying in a road traffic crash & 3.2 & 2.7 \\
\hline
\end{tabular}

* All comparisons were statistically significant: $p<0.01, \chi^{2}$ test standardized death rates based on the WHO population indicate an expected mortality rate of 7.9 per 10,000 in Kampala compared to 4.1 in SF and 5.5 in the US, which indicates that the comparative injury mortality ratio in Kampala is $93 \%$ higher than in SF and $44 \%$ higher than in the US after controlling for age (Table 4).

Cause-specific deaths

The most commonly recorded causes of injury in Kampala were road traffic crashes (46\%), assaults (16\%), and burns $(9 \%)$, although cause-specific data were not available in $21 \%$ of injury deaths. Road traffic crashes, including pedestrian injury, had the highest rate of injury (3.1 deaths/ 10,000 people) in Kampala. In the US as a whole, road traffic crashes resulted in $37 \%$ of all injury deaths. This was followed by poisoning (20\%) and falls (17\%). In San Francisco, assault, mostly homicide, led the causes of injury deaths (38\%). Road crashes (36\%) and falls (18\%) ranked second and third. In this database, the odds of dying from a road traffic crash were 2.4 times higher in Kampala than in SF and 2.3 times higher than in the US.

\section{Body region most affected}

In Kampala, the most common body regions associated with injury-related death were the head and neck in $65 \%$ $(n=528)$, followed by the abdomen $(20 \%, n=162)$, chest $(19 \%, n=151)$, and extremities $(18 \%, n=148)$. Injuries to multiple body regions were recorded in $91 \%$ of cases. Body region could not be classified in $17 \%$ of records. In the SF data the proportion of injury deaths with head and neck injury was $34 \%$; and in the US data it was $28 \%$. The odds of dying with a head and/or neck injury in Kampala were 3.7 times greater than in SF and 4.8 times greater than in the US.

Table 4 Distribution of injury-related deaths in the two cities

\begin{tabular}{lll}
\hline Injury-related deaths & $\begin{array}{l}\text { Kampala } \\
\text { (no.) }\end{array}$ & $\begin{array}{l}\text { San Francisco } \\
\text { (no.) }\end{array}$ \\
\hline Total injury-related deaths & 812 & 357 \\
Deaths in the prehospital setting & $434(53 \%)$ & $243(68 \%)$ \\
Deaths at a hospital & $378(47 \%)$ & $114(32 \%)$ \\
At Mulago Hospital or SFGH & $370(98 \%)$ & $114(100 \%)$ \\
At another hospital & $8(2 \%)$ & 0 \\
Of injury deaths at Mulago Hospital & 370 & 114 \\
or SFGH & & \\
$\begin{array}{l}\text { Death in emergency department } \\
\text { Death in the inpatient ward }\end{array}$ & $23(6 \%)$ & $40(35 \%)$ \\
\end{tabular}

SFGH San Francisco General Hospital 


\section{Discussion}

This study shows that injury-related mortality is high in urban Uganda and that injuries result in $25 \%$ of all recorded deaths in Kampala. The odds of dying due to injury are five times higher in Kampala than in San Francisco or the United States. Road traffic injuries accounted for $46 \%$ of injury-related deaths in Kampala, and injury to the head and neck was a commonly implicated cause of death.

Our study's injury mortality rate of $6.7 / 10,000$ is much lower than the $22 / 10,000$ previously reported by community surveys in urban Kampala [20]. One reason for this difference may be overestimation in the previous study due to recall or interviewer bias. In addition, the previous study was conducted in only one political district in Kampala and may not have been representative of the entire city. Our study included deaths recorded in all of Kampala except any undocumented burials or deaths not reported to the Kamala City Council Mortuary by the private and mission hospitals in the city. Moreover, our study was not affected by recall or interviewer bias. Thus, our results may more accurately describe the overall urban injury mortality in Kampala.

Our findings can be compared with injury-related mortality reports from other countries in Africa. In Kumasi, Ghana, injuries were implicated in $8.6 \%$ of all deaths recorded in 1996 [11]. However, this estimate may have been low as the study included only one mortuary in the city; immediate burials without notification of medical facilities, mortuaries, or the country's Birth and Death Registry may have occurred. Additionally, our much higher injury mortality rate in Kampala may be due to rapid increases in the burden of injuries and urban population density in Uganda since 1996. Between 1985 and 1995 alone, the number of licensed motor vehicles in Uganda increased by $220 \%$, and the city's population increased by 4-5\% per year [15]. These rapid changes may be contributing to a much greater role of injury as a cause of death in Kampala. In fact, more recent hospital-based analyses of injury mortality from Nigeria have reported findings similar to ours $[19,20]$. Furthermore, as many as $81 \%$ of injuryrelated deaths in Kumasi have been reported to occur in the prehospital setting [11], unlike our findings in Kampala, where $53 \%$ of all injury deaths took place outside the hospital. This difference may be explained by differences in types of injury, the mechanism or injury severity seen in the two settings, or the on-scene care provided by an informal network of first responders in Kampala [21].

Although studies on emergency department visits for injury have been conducted in Kampala [17, 22] ours is the first study to describe the injury-related mortality patterns in detail using a new, comprehensive death statistics database. It also provides a comparison with the more organized trauma system in San Francisco. Our findings raise several important concerns about the etiology of injury in this setting, the availability of appropriate prevention measures, prehospital and trauma care, and the need for organized data collection. In addition, our study provides further evidence of the tremendously high burden of injury in a developing country.

First, the high prevalence of road traffic crashes and injury to the head and neck in Kampala must be explored further to generate specific policy responses. Improving road safety involves a multisectoral approach with buy-in from law enforcement agencies, road users, and city planners. Traffic safety enforcement has been suggested as a cost-effective method of addressing the injury epidemic in Kampala [23]. The Kampala Police Department and the Injury Control Center, Uganda have led ongoing advocacy for helmet use by motorcycle drivers and passengers. Increased rates of helmet use may be a valuable component when addressing the high injury-related mortality in Kampala, particularly the high proportion of traumatic deaths that include injury to the head and neck.

Second, the lack of a formal emergency medical system in Kampala is an important issue. Immediate prehospital care by trained first responders has been shown to save lives in other settings [22, 23]. Adequate airway protection and hemorrhage control alone could potentially salvage many of those with head injury who may otherwise die in the prehospital setting. Mechanisms to formalize prehospital care in Kampala, including training lay first responders in basic prehospital trauma care, are being explored and may be one approach to addressing injuryrelated mortality [21]. Furthermore, improving hospitalbased trauma care by implementing context-specific trauma training programs for nurses and doctors may ensure that injury victims who reach the hospital receive fast and appropriate care. Such programs have reduced injury mortality in other settings [24]. Because $47 \%$ of injury-related deaths in Kampala $(n=347)$ occurred at the hospital level, compared to only $21 \%$ in San Francisco ( $n=74)$, optimizing in-hospital trauma care may be a key area for policy change to reduce the burden of injury in Kampala. Additionally, engaging in such capacity-building interventions for trauma and emergency surgical services across the spectrum of care in Kampala may improve medical services for other emergency conditions. Thus, these programs could strengthen the health system broadly compared to other vertical, disease-specific programs.

Third, another critical issue raised by our study is the need for accurate injury-related vital statistics. One to two million deaths occurring annually in low- and middleincome countries are potentially avertable with better trauma systems [9]. It is unclear how many injury-related deaths in Kampala are avertable today; a prospective vital statistics database would help track injury deaths and 
determine the deaths that are avoidable. Resource-rich countries have begun to understand the importance of investing in injury prevention, relative to other causes; and there is increasing evidence that such investments are even more necessary in low- and middle-income countries such as Uganda. A vital statistics database could also help evaluate essential interventions for injury prevention and trauma care. For example, the WHO and the World Bank have recommended a lay first-responder system in settings where formal emergency services do not exist. Preliminary results of a pilot first-responder program in Kampala have been reported elsewhere [21, 25]. However, the impact of such a program on injury-related mortality can only be evaluated with adequate infrastructure to capture vital statistics.

Our study has several limitations. First, we may not have captured all injury deaths in urban Kampala because although Mulago Hospital serves as the sole 24-hour public emergency department in the city and sees $75 \%$ of all injury victims in Kampala [17] deaths occurring either at home or en route to any of the city's other hospitals may not have been recorded accurately. Although we assumed that most of these deaths would have been reported to the Kampala City Council Mortuary, some of these deaths may have been missed.

Second, the three data sources in Kampala collect data based on the occurrence of death within the city and not specifically the residence of the decedent. Residents who died outside the city would not be captured by the sources. Likewise, nonresidents of Kampala may be incorrectly assigned a local address. Thus, the records may have overestimated or underestimated deaths of Kampala residents. We assumed that these figures are either negligible or balance each other out. However there can be considerable movement of people between Kampala and the rest of country at the end of the year, when families travel for the holiday season, leading to an increase in the incidence of injuries, although no seasonal variations in injury deaths were apparent in our data. The estimates of the population at risk may also be inaccurate, although this issue exists in all registries. Finally, data quality may be an issue. For example, the records of deaths from medical causes may underestimate the contributions of infectious disease because of diagnostic challenges in the postmortem setting.

Third, the validity of comparing Kampala and San Francisco depends on intrinsic comparability of both cities. Although we accounted for differences in demographics through direct standardization, each city varies from the other in regard to cultural norms, road infrastructure, and traffic safety regulations. These issues may influence injury-related mortality rates in different ways in each setting, making this comparison less valid. For example, prehospital injury-related deaths occur at a higher rate in
San Francisco than in Kampala possibly due to the high incidence of assault, particularly homicide, which may be resulting in severe nonsurvivable penetrating injury in the U.S. setting. Therefore, controlling for injury severity may improve the accuracy of our findings. Nevertheless, the two cities may have comparable injury patterns based on key similarities such as high pedestrian traffic, reliance on public transportation, and provision of definitive trauma care at a single city hospital.

Despite these limitations, our study contributes to a better understanding of the burden of injuries in a resourceconstrained setting. Many other issues related to injury still have to be addressed. The disability associated with injury is a substantial burden on society $[2,25]$ and needs to be further explored in this setting. An economic evaluation of the high injury-related mortality rate in Uganda would also add to a greater understanding of the true societal cost of this epidemic and better inform resource allocation.

\section{Conclusions}

The urban injury-related mortality rate is significantly higher in Kampala than in the United States and San Francisco. Rates are highest among young adults, suggesting that Uganda faces an enormous economic burden from this problem. Context-specific interventions are urgently needed to address this underappreciated impact of trauma, especially the high prevalence of head and neck injuries. Better access to emergency services and stronger injury prevention and control policies in Uganda could help address these substantial inequities and reduce needless mortality and morbidity.

Acknowledgments We are grateful for the support of the staff from the Mulago Hospital Medical Records Department, Kampala City Council Mortuary and the Ministry of Health's Government Chemist Office for allowing us to work with the various data sets. Our thanks also go to Dr. Charles Mock of the WHO for his critical review of the manuscript and University of California San Francisco's Department of Surgery Scientific Publications Manager, Pamela Derish, for her editorial support. Funding Sources included The Laura Case Trust and the Hellman Family Foundation.

Conflicts of interest The authors have no conflicts of interest.

Open Access This article is distributed under the terms of the Creative Commons Attribution Noncommercial License which permits any noncommercial use, distribution, and reproduction in any medium, provided the original author(s) and source are credited.

\section{References}

1. WHO (2005) Prehospital Trauma Care Systems. WHO, Geneva

2. WHO (2004) World report on road traffic injury prevention. WHO, Geneva 
3. Nantulya VM, Reich MR (2003) Equity dimensions of road traffic injuries in low- and middle-income countries. Inj Control Saf Promot 10(1-2):13-20

4. WHO (2008) The global burden of disease: 2004 update. WHO, Geneva

5. Bhalla K, Harrison J, Abraham J et al (2009) Data sources for improving estimates of the global burden of injuries: call for contributors. PLoS Med 6(1):e1

6. Bhalla K, Naghavi M, Shahraz S et al (2009) Building national estimates of the burden of road traffic injuries in developing countries from all available data sources: Iran. Inj Prev 15: $150-156$

7. Setel PW, Macfarlane SB, Szreter S et al (2007) A scandal of invisibility: making everyone count by counting everyone. Lancet 370:1569-1577

8. Hofman K, Primack A, Keusch G et al (2005) Addressing the growing burden of trauma and injury in low- and middle-income countries. Am J Public Health 95:13-17

9. Mock CN, Jurkovich GJ, nii-Amon-Kotel D et al (1998) Trauma mortality patterns in three nations at different economic levels: implications for global trauma system development. J Trauma 44:804-812 discussion 812-814

10. Mathers CD, Bernard C, Iburg KM et al (2003) Global burden of disease in 2002: data sources, methods and results. Global programme on evidence for health policy discussion paper no. 54 . WHO, Geneva

11. London J, Mock C, Abantanga FA et al (2002) Using mortuary statistics in the development of an injury surveillance system in Ghana. Bull WHO 80:357-364

12. Mock CN, nii-Amon-Kotei D, Maier RV (1997) Low utilization of formal medical services by injured persons in a developing nation: health service data underestimate the importance of trauma. J Trauma 42:504-511 discussion 511-513

13. WHO (2009) WHO Statistical Information System (WHOSIS). Accessed June 8, 2009. WHO, Geneva

14. Anonymous (2009) Key Development Data \& Statistics. Accessed June 8, 2009. World Bank, Washington, DC
15. Anonymous. Kampala City Council District Profile. http://www. citycouncilofkampala.go.ug/downloads/district_profile.pdf/

16. Anonymous (2006) Vital Statistics Query System Online. Cited March 18, 2009. Center for Health Statistics, California Department of Public Health, http://www.applications.dhs.ca.gov/vsq/ default.asp/

17. Kobusingye OC, Guwatudde D, Owor G et al (2002) Citywide trauma experience in Kampala, Uganda: a call for intervention. Inj Prev 8:133-136

18. Anonymous (2005) Web-based Injury Statistics Query and Reporting System (WISQARS) Online. http://webappa.cdc.gov/ sasweb/ncipc/mortrate10_sy.html. Cited March 18, 2009. Centers for Disease Control and Prevention, National Center for Injury Prevention and Control

19. Ahmad OB, Boschi-Pinto C, Lopez AD et al (2001) Age standardization of rates: a new WHO standard. WHO, Geneva

20. Kobusingye O, Guwatudde D, Lett R (2001) Injury patterns in rural and urban Uganda. Inj Prev 7:46-50

21. Jayaraman S, Mabweijano JR, Lipnick MS et al (2009) Current patterns of prehospital trauma care in Kampala, Uganda and the feasibility of a lay-first-responder training program. World J Surg 33:2512-2521

22. Kobusingye OC, Lett RR (2000) Hospital-based trauma registries in Uganda. J Trauma 48:498-502

23. Bishai D, Asimwe B, Abbas S et al (2008) Cost-effectiveness of traffic enforcement: case study from Uganda. Inj Prev 14: 223-227

24. Ali J, Adam R, Butler AK et al (1993) Trauma outcome improves following the advanced trauma life support program in a developing country. J Trauma 34:890-898 discussion 898-899

25. Jayaraman S, Mabweijano JR, Lipnick MS et al (2009) First things first: effectiveness and scalability of a basic prehospital trauma care program for lay first-responders in Kampala, Uganda. PLoS One 4:e6995 